\title{
Object Extraction in Gray-Scale Images by Optimizing Roughness Measure of a Fuzzy Set
}

\author{
D.V. Janardhan Rao, Mohua Banerjee, and Pabitra Mitra \\ Indian Institute of Technology Kanpur, \\ Kanpur 208016, India \\ \{dvjrao, pmitra, mohua\}@iitk.ac.in
}

\begin{abstract}
Object extraction from gray-tone images involve handling of inherent uncertainties in an image. Traditionally fuzzy set theoretic techniques are used for this purpose. However, roughness and limited discernibility of objects is another important aspect of image uncertainty. In this article we propose an algorithm for selection of intensity threshold for object extraction by optimizing a roughness measure of the fuzzy set corresponding to the image object. The rough-fuzzy algorithm is tested on some benchmark images.
\end{abstract}

\section{Introduction}

The theory of Rough sets [1] has emerged as a major mathematical approach for managing uncertainty that arises from inexact, noisy, or incomplete information. The focus of rough set theory is on the ambiguity caused by limited discernibility of objects in the domain of discourse. The idea is to approximate any concept (a crisp subset of the domain) by a pair of exact sets, called the lower and upper approximations. On the other hand, fuzzy set theory hinges on the notion of a membership function on the domain of discourse, assigning to each object a grade of belongingness in order to represent an imprecise property. In practice, uncertainties of both the above type appear in nature, and it is apt to characterize both of them. Toward this goal several roughness measures of fuzzy sets (and vice versa) has been defined in literature [23]. The objective being to obtain more flexible representation of imprecise objects.

Object extraction refers to the task of segmenting the target image from its background. It is useful in a number of image processing and computer vision tasks. Images are inherently gray and of imprecise nature and can be modeled as a fuzzy set. The main challenge in object extraction from gray-tone images is that of handling uncertainties owing to grayness of the object boundaries. In literature several fuzzy set theoretic techniques has been proposed for this purpose. A review of these approaches is provided in [4]. Some of the poupular ones are based on the principle of minimizing fuzzy entropy [5]. It may be noted that probabilistic Shannon entropy can also be used for object extraction from gray-scale images [6].

Human vision system perceives objects only up to a limited (often variable and multiscale) spatial (and intensity) resolution or discernibility. It is expected 
that rough set representation of image objects in terms its lower and upper approximation would be beneficial in handling the image uncertainties in the border region of object and background. In this article we model an image as a rough-fuzzy set and use a 'roughness of a fuzzy set' measure to determine the gray-level threshold for segmenting an gray-tone object from its background.

The proposed approach for object extraction in gray-tone images comprises of the following steps. First we calculate the histogram of an image $X$ and define a fuzzy object region over the image space with membership plane $A=\mu_{X}$ using a one-dimensional $\pi$-function with constant bandwidth. We control the thresholds $\alpha$ and $\beta$ (which gives the lower and upper approximation of the fuzzy object region respectively) to minimize the roughness measure of the fuzzy object region. The threshold value is selected based on $\beta$ for which roughness is minimum.

\section{Rough Sets and Rough-Fuzzy Sets}

We first define some basic concepts of rough set theory necessary for explanation of the object extraction algorithm. Let the domain $U$ of discourse (also called universe) be a non-empty finite set, and $R$ an equivalence relation on $U$. The pair $\langle U, R\rangle$ is called an approximation space [1. Let $X_{1}, \ldots, X_{n}$ denote the equivalence classes in $U$ due to $R$, i.e., $\left\{X_{1}, \ldots, X_{n}\right\}$ forms a partition of $U$. If $A \subseteq U$, the lower approximation $\underline{A}$ and upper approximation $\bar{A}$ of $A$ in the approximation space $\langle U, R>$ are respectively given as follows [1]:

$\underline{A}=\cup\left\{X_{i} / X_{i} \subseteq A\right\}$ and $\bar{A}=\cup\left\{X_{i} / X_{i} \cap A \neq \phi\right\}$, where $i \in\{1, \ldots, n\}$

$\underline{A}(\bar{A})$ is interpreted as the collection of those objects of the domain $U$ that definitely (possibly) belong to $A$. The pair $\langle\underline{A}, \bar{A}\rangle$ may be called the rough approximation of the set $A$ or simply rough set.

Roughness of a set $A$ in the approximation space $\langle U, R\rangle$ is reflected by the ratio of the number of objects in its lower approximation to that in its upper approximation - the greater the value of the ratio, the lower the roughness. More explicitly, a measure $\rho_{A}$ of roughness of $A$ in $\langle U, R\rangle$ is defined thus [1]:

$$
\rho_{A}=1-\frac{|\underline{A}|}{|\bar{A}|},
$$

where $|X|$ denotes the cardinality of a set $X$.

We next come to the definitions of a rough fuzzy set and allied notions [3], roughness measure of a fuzzy set [2, that shall form the basis of this work. Let $A: U \rightarrow[0,1]$ be a fuzzy set in $U, A(x), x \in U$, giving the degree of membership of $\mathrm{x}$ in $\mathrm{A}$.

Definition 2.1: The lower and upper approximations of the fuzzy set $A$ in $U$, denoted $\underline{A}$ and $\bar{A}$, respectively, are defined as fuzzy sets in $U / R\left(=\left\{X_{1}, \ldots, X_{n}\right\}\right)$, i.e., $\underline{A}, \bar{A}: U / R \rightarrow[0,1]$, such that $\underline{A}\left(X_{i}\right)=\operatorname{Min}_{x \in X_{i}} A(x)$ and $\bar{A}\left(X_{i}\right)=$ $\operatorname{Max}_{x \in X_{i}} A(x)$, where $i=1, \ldots, n<\underline{A}, \overline{\bar{A}}>$ is called a rough fuzzy set. 
Definition 2.2: Fuzzy sets $\underline{F}$ and $\bar{F}: \mathrm{U} \rightarrow[0,1]$ are defined as follows: $\underline{F}(x)=\underline{A}\left(X_{i}\right)$ and $\bar{F}(x)=\bar{A}\left(X_{i}\right)$ if $x \in X_{i}, i \in\{1, \ldots, n\}$,

$\underline{F}$ and $\bar{F}$ are fuzzy sets with constant membership on the equivalence classes of $\bar{U}$. For any $x$ in $U, \underline{F}(x)(\bar{F}(x))$ can be viewed as the degree to which $x$ definitely (possibly) belongs to the fuzzy set $A$.

\section{Roughness Measure of a Fuzzy Set}

In this section, we define the roughness measure of a fuzzy set 2 and state some properties and observations on this measure which are used in the present work. Let $\alpha, \beta$ be two parameters, where $0<\beta \leq \alpha \leq 1$. Consider the $\alpha$-cut $\underline{F}_{\alpha}, \beta$-cut $\bar{F}_{\beta}$ of the fuzzy sets $\underline{F}, \bar{F}$ respectively. $\underline{F}_{\alpha}=\{x / \underline{F}(x) \geq \alpha\}$ and $\overline{\bar{F}}_{\beta}^{\alpha}=\{x / \bar{F}(x) \geq \beta\}$.

It can then be said that $\underline{F}_{\alpha}\left(\bar{F}_{\beta}\right)$ is the collection of objects in $U$ with $\alpha(\beta)$ as the minimum degree of definite (possible) membership in the fuzzy set $A$. In other-words, $\alpha, \beta$ act as thresholds of definiteness and possibility, respectively, in membership of the objects of $U$ to $A . \underline{F}_{\alpha}, \bar{F}_{\beta}$ are called $\alpha$-lower approximation and $\beta$-upper approximation of the fuzzy set $A$ in $\left\langle U, R>. \underline{F}_{\alpha}=\cup\left\{X_{i} / X_{i} \in\right.\right.$ $\left.\underline{A}_{\alpha}\right\}$ and $\underline{F}_{\beta}=\cup\left\{X_{i} / X_{i} \in \bar{A}_{\beta}\right\}$, where $i=\{1, \ldots, n\}$ and $\underline{F}_{\alpha}, \bar{F}_{\beta}$ are the $\alpha$ - and $\beta$-cuts, respectively of the fuzzy sets $\underline{A}$ and $\bar{A}$ (cf. Definition 2.1). So, alternatively, $\underline{F}_{\alpha}\left(\bar{F}_{\beta}\right)$ can be looked upon as the union of those equivalence classes of $U$ that have degree of membership in the lower (upper) approximation $\underline{A}(\bar{A})$ of $A$ at least $\alpha(\beta)$. Since $\alpha \geq \beta, \underline{F}_{\alpha} \subseteq \bar{F}_{\beta}$.

Definition 3.1: The roughness measure $\rho_{A}^{\alpha, \beta}$ of the fuzzy sets $A$ in $U$ with respect to parameters $\alpha, \beta$ where $0<\beta \leq \alpha \leq 1$, and the approximation space $<U, R>$ is defined as:

$$
\rho_{A}^{\alpha, \beta}=1-\frac{\left|\underline{F}_{\alpha}\right|}{\mid \bar{F}} \beta \mid
$$

Lemma 3.1: (a) If $\beta$ is kept fixed and $\alpha$ increased $\left|\underline{F}_{\alpha}\right|$ decreases and $\rho_{A}^{\alpha, \beta}$ increases.
(b) If $\alpha$ is kept fixed and $\beta$ increased, $\left|\bar{F}_{\beta}\right|$ decreases and $\rho_{A}^{\alpha, \beta}$ decreases.
(c) If $A \subseteq B$ and $\bar{F}_{\beta}^{A}=\bar{F}_{\beta}^{B}$, then $\rho_{B}^{\alpha, \beta} \leq \rho_{A}^{\alpha, \beta}$.
(d) If $A \subseteq B$ and $\bar{F}_{\alpha}^{A}=\bar{F}_{\alpha}^{B}$, then $\rho_{A}^{\alpha, \beta} \leq \rho_{B}^{\alpha, \beta}$. These follow from the properties of rough and fuzzy sets.

\section{Application to Object Extraction in Gray-Scale Images}

The roughness measure $\rho$ has many applications in pattern recognition and image analysis problems. Let $X$ denote a gray-tone image or feature space, and $X_{1}, \ldots, X_{n}$ represent $n$ regions. The fuzzy set $A$ can be viewed to represent the ill-defined pattern classes or some imprecise image property such as brightness, darkness, edginess, smoothness etc. Relative to thresholds $\alpha, \beta$ roughness of such 
an imprecise property $A$ can then be measured in terms of the ratio of number of feature points definitely satisfying $A$ to the number of feature points possibly satisfying $A$. The concept of splitting $X_{1}, \ldots, X_{n}$ is analogous to increasing the resolution of a digital image, and can well be utilized for sub-pixel classification problems and for detecting the boundaries of regions precisely.

The algorithm for object extraction in gray-tone images using the roughness measure $\rho$ is described below.

\section{Algorithm}

Step 1: Compute the histogram of the gray-tone image $X$ and define a fuzzy object region over the image space with membership plane $A=\mu_{X}$. The onedimensional $\pi$-function, with range [0,1], and constant bandwidth may be used as a membership function. It is represented as:

$$
\pi\left(f_{i j}, c, \lambda\right)= \begin{cases}2\left(1-\frac{\left\|f_{i j}-c\right\|}{\lambda}\right)^{2} & \text { for } \frac{\lambda}{2} \leq\left\|f_{i j}-c\right\| \leq \lambda \\ 1-2\left(\frac{\left\|f_{i j}-c\right\|}{\lambda}\right)^{2} & \text { for } 0 \leq\left\|f_{i j}-c\right\| \leq \frac{\lambda}{2} \\ 0 & \text { otherwise }\end{cases}
$$

where $\lambda(>0)$ is the radius of the $\pi$-function with $c$ as central point.

We use the $\pi$-function as it is a general form which can present both Sfunction by the left part and the inverse of S-function by the right part. Therefore, the regions at the lowest and highest ends of features can also be represented by $\pi$ functions.

Step 2: Partition the image $X$ into $X_{1}, \ldots, X_{n}$, regions such that each $X_{i}$ is a $w \times w$ pixel non-overlapping window of the image. Note that, any sub-image of $X$ based on an arbitrary equivalence relation $R$ may be used. The choice of $w$ is determined by factors like amount of imprecision and computational complexity. We have done our experiments for different values of $w$.

Step 3: Vary the thresholds $\alpha$ and $\beta$ which determine the lower and upper approximations according to Lemma 3.1. Determine $\alpha$ and $\beta(\alpha>\beta)$ which minimises the roughness measure $\rho$ (Equation 2) of the fuzzy object region $A$.

Step 4: Threshold the gray-tone image using $\beta$ corresponding to minimum value of $\rho$. Note that, in conventional fuzzy segmentation, threshold is determined using only class membership information of pixels in the $\mu_{X}$-plane. Here, in addition the lower and upper approximations of the $\mu_{X}$-plane are taken into consideration.

Alternately, one can define a fuzzy object region over the image space with membership plane $A=\mu_{X}$ of constant bandwidth using a two-dimensional $\pi$ function. Vary the cross-over point of $\mu_{X}$-plane and compute $\rho$ for a fixed value of $\alpha$ and $\beta$. Find that $\mu_{X}$-plane for which $\rho$ is minimum. Such a $\mu_{X}-$ plane represents the fuzzy segmented version of the image (with lower and upper approximations as determined by $\alpha$ and $\beta$ ). 


\section{Experimental Results}

The rough-fuzzy object extraction algorithm was tested on several images of different types. Figure 1, shows the extracted objects for the image of an airplane. The image contain objects with gray boundaries. Results are presented for thresholds obtained by optimizing probabilistic Shannon entropy [6] and the proposed rough-fuzzy uncertainty measure. The rough-fuzzy entropy measure was computed using granule sizes of $4 \times 4$ aqnd $8 \times 8$ respectively. It can be seen from the figures that the rough-fuzzy algorithm has extracted the objects with a high accuracy. It also outperforms the method based on probabilistic Shannon's entropy.

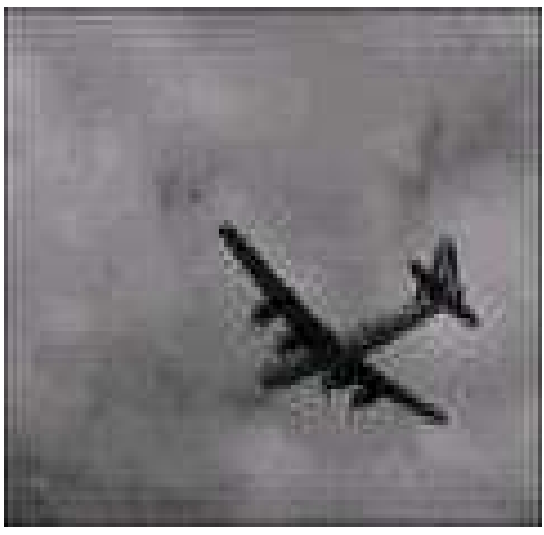

(a)

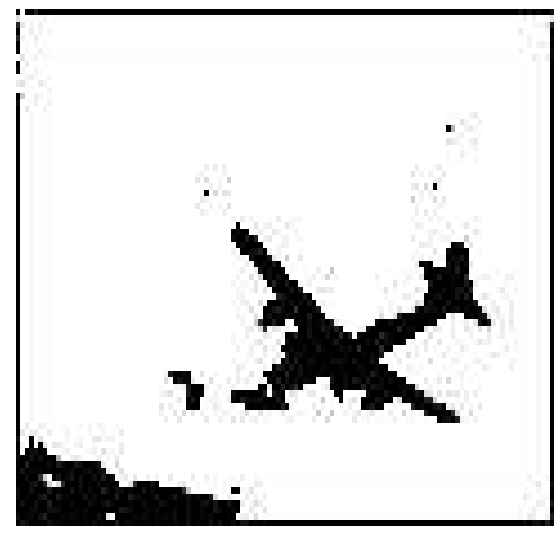

(c)

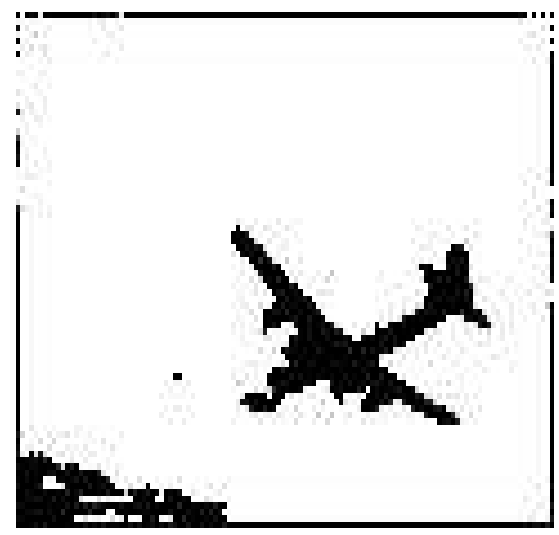

(b)

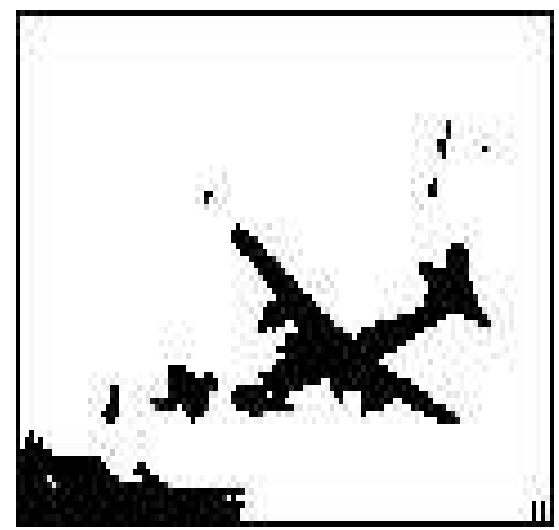

(d)

Fig. 1. Airplane image: (a) original image, and segmented images using (b) roughfuzzy measure with $4 \times 4$ pixel granules, and (c) rough-fuzzy measure with $8 \times 8$ pixel granules, (d) Shannon entropy 


\section{Conclusions and Discussion}

In this article we propose a method for object extraction in gray-scale images based on roughness measure of a fuzzy set.In conventional fuzzy segmentation, uncertainty is handled in terms of only class membership of pixels in the $\mu_{X}$ -plane. Here, in addition, the lower and upper approximations of the $\mu_{X}$-plane are taken into consideration for managing uncertainty. Our future work would be to use this roughness measure for some other pattern recognition and image analysis problems like image enhancement and sub-pixel classification.

\section{References}

1. Pawlak, Z.: Rough sets : Theoretical Aspects of Reasoning about Data. Kluwer Academic (1991)

2. Banerjee, M., Pal, S.: Roughness of a fuzzy set. Information Sciences 17 (1996) 235-246

3. Dubois, D., Prade, H.: Rough fuzzy sets and fuzzy rough sets. Int.J.General Syst 17 (1990) 191-298

4. Pal, N., Pal, S.: A review on image segmentation techniques. Pattern Recognition 26 (1993) 1277-1294

5. Ruberto, C.D., Nappi, M., Vitulano, S.: Image segmentation by means of fuzzy entropy measure. In: ICIAP '97: Proc. 9th Intl Conf Image Analysis and ProcessingVolume I, London, UK, Springer-Verlag (1997) 214-222

6. Kapoor, J., Sahoo, P., Wong, A.: A new method for gray-level picture thresholding using the entropy of the histogram. Comput. Vision Graphics Image Process 29 (1985) 273-285 